\title{
Removal of Respiratory Influences from Heart Rate During Emotional Stress
}

\author{
Carolina Varon ${ }^{1,2}$, Jesús Lázaro ${ }^{1,2}$, Alberto Hernando ${ }^{3}$, Alexander Caicedo ${ }^{1,2}$, \\ Sabine Van Huffel ${ }^{1,2}$, Raquel Bailón ${ }^{4}$ \\ ${ }^{1}$ KU Leuven, Department of Electrical Engineering (ESAT), STADIUS Center for Dynamical \\ Systems, Signal Processing and Data Analytics, Leuven, Belgium \\ ${ }^{2}$ imec, Leuven, Belgium \\ ${ }^{3}$ Centro Universitario de la Defensa, Zaragoza, Spain \\ ${ }^{4}$ BSICoS Group, Aragón Institute of Engineering Research (I3A), IISAragon, University of Zaragoza \\ and CIBER-BBN, Zaragoza, Spain
}

\begin{abstract}
Heart rate variability $(H R V)$ has been proposed as an indicator of stress. However, respiratory changes affect the spectral content of the HRV, resulting in a misleading estimation of stress, especially when the respiratory rate falls into the classical low frequency band. To overcome this limitation of the classical HRV analysis, this study decomposes the HRV signal, recorded during different phases of acute emotional stress, into two components using orthogonal subspace projections (OSP). One component describes all linear respiratory influences, and the other one contains all residual HRV dynamics. Two subspace definitions are compared here, on the one hand, the original respiration signal, and on the other hand, its wavelet decomposition. After a multicomparison test, no difference was found between the respiratory components derived using both subspaces, hence, no added value is achieved by the wavelet decomposition. Furthermore, the HRV variations that are linearly related to respiration are significantly different $(p<0.008)$ between relax and emotional stress. This suggests that respiratory dynamics are enough to detect emotional stress, which might result in an improved assessment of stress.
\end{abstract}

\section{Introduction}

The reliability of the classical heart rate variability (HRV) analysis strongly depends on the respiratory rate. For instance, the respiratory rate is not always within the high frequency (HF) band of HRV. Instead, it might fall into the low frequency (LF) band, causing an overestimation of the sympathetic activity of the autonomic nervous system (ANS). This is often the case during periods of relaxation, as reported in [1] and [2]. A different scenario where misleading results can be obtained is when the res- piratory rate exceeds the upper limit of HF. This can occur during some periods of emotional stress and it can result in an underestimation of the parasympathetic activation of the ANS [1]. To overcome this limitation, a correction of the HF band can be applied based on the respiratory rate, as used in [1]. This adaptation allowed to detect differences between stress phases in both LF and HF bands, which can be associated with a better quantification of the ANS modulation. However, this adaptation can only be implemented when the respiratory signals have a peaked power spectra.

A different way to tackle the limitations of the classical HRV is by removing respiratory influences from the heart rate and analysing the residual dynamics, as done in [2] for the analysis of mental stress, and in [3] for the detection of sleep apnea. This can be achieved by means of orthogonal subspace projections (OSP), where the heart rate is first projected onto a respiratory subspace, and then decomposed into two different components. One component related to all linear respiratory influences, and one component describing all residual dynamics of heart rate. The respiratory subspace was defined in [2] using the wavelet decomposition of the respiratory signal, and in [3] using the original respiratory signal. In this study, both subspace definitions will be used to study the respiratory interactions during acute emotional stress, and the added value of the wavelet decomposition will be evaluated. Furthermore, the frequency content of the different heart rate components will be analyzed for different stress phases. As a result, the influence of respiration on the HRV during emotional stress will be assessed.

\section{Methodology}

\subsection{Data}

The dataset used in this study consists of ECG and respiratory signals recorded at the Autonomous University of 
Barcelona (UAB) and the University of Zaragoza (UZ), from 46 volunteers, 18 men and 28 women. The ECG signals were sampled at $1000 \mathrm{~Hz}$ and the respiratory signals at $250 \mathrm{~Hz}$. The mean age of the volunteers was $21.76 \pm 4.48$ years, and they all underwent a stress session. During this session, emotional stress was induced by means of a modified Trier Social Stress Test [1], which includes the following phases:

- A baseline (BL) of about 10 minutes when the subject listens to a relaxing audio

- Story telling (ST): 3 stories are told to the subject and he/she is asked to remember as many details as possible.

- Memory task (MT): The subject is asked to tell in front of a camera, all details that he/she remembered from the ST phase.

- Stress anticipation (SA): The subject is asked to wait for about 10 minutes for the results of the evaluation of the MT phase.

- Video exposition (VE): The video recorded during the MT phase is shown to the subject together with another video, where an actor repeats the stories in a perfect way. The idea is to make a comparison between the "poor" performance of the subject and a much better performance of the actor.

Eight subjects did not complete the test, and two had low ECG signal quality in some phases, hence, their recorded data were removed from the dataset. Furthermore, the MT phase was also removed due to, in some cases, short term duration $(\approx 30 s)$ and low signal quality since the subjects were speaking during this phase. In total, 36 subjects were analyzed and the average duration of each remaining phase was as follows. BL: $11.5 \pm 1.4$ minutes; ST: $1.66 \pm 0.5 \mathrm{~min}-$ utes; SA: $11.1 \pm 1.2$ minutes; VE: $3.1 \pm 0.48$ minutes.

\subsection{Pre-Processing}

The respiratory signals were first band-pass filtered using a Butterworth filter with cutoff frequencies at 0.03 and $0.9 \mathrm{~Hz}$. Then, they were downsampled at $4 \mathrm{~Hz}$, and normalized with zero mean and unit variance.

The ECG recordings were used to derive the HRV signals from the heart beats occurrence time series. The latter were detected using the algorithm presented in [3], and ectopic beats, missed peaks and false detections were corrected using the integral pulse frequency modulation (IPFM) model [4]. The resultant time series were further corrected for variations in the mean heart rate, and the HRV signals were then derived as in [1]. Finally, the HRV signals were resampled at $4 \mathrm{~Hz}$, filtered and normalized, similar to the respiratory signals.

In order to avoid transient behavior between stress phases, the first and last seconds of the signals were removed from the analysis. For BL and SA 1 minute, for ST 5 seconds, and for VE 10 seconds.

\subsection{Heart Rate Decomposition}

Given are two physiological signals: the respiratory signal denoted by $\mathbf{X}$ and the HRV signal denoted by $\mathbf{Y}$. All the information contained in $\mathbf{Y}$ that is linearly related to $\mathbf{X}$, can be computed by projecting $\mathbf{Y}$ onto a subspace $\mathbb{V}$ defined by variations in $\mathbf{X}$. One way of constructing the subspace related to respiration is by using the original $\mathbf{X}$ and its delayed versions to create the basis $\mathbf{V}_{s}$, using delays from 1 to $m$ seconds [3]. This subspace will be denoted by $\mathbb{V}_{s}$.

The respiratory subspace can also be constructed using the wavelet decomposition of $\mathbf{X}$ with $d b 4$ as a mother wavelet and 5 levels of decomposition, as done in [2]. The detail coefficients and their delayed versions up to $m$ seconds are then used to define the basis $\mathbf{V}_{w}$ of the subspace $\mathbb{V}_{w}$. In order to compare the performance of both approaches, namely, the decomposition using two different subspaces $\mathbb{V}_{s}$ and $\mathbb{V}_{w}$, delays of up to 3 seconds (i.e., $m=3$ ) are used [2].

After constructing a basis $\mathbf{V}$ for the subspace $\mathbb{V}$, any signal $\mathbf{Y}$ can be projected onto the subspace, by means of

$$
\mathbf{Y}_{\mathbf{X}}=\mathbf{P Y}
$$

with $\mathbf{P}$ a projection matrix defined as

$$
\mathbf{P}=\mathbf{V}\left(\mathbf{V}^{\mathbf{T}} \mathbf{V}\right)^{-\mathbf{1}} \mathbf{V}^{\mathbf{T}} .
$$

Note that $\mathbf{Y}_{\mathbf{X}}$ describes the dynamics of $\mathbf{Y}$ related to $\mathbf{X}$, and the part of $\mathbf{Y}$ that is related to other mechanisms can be computed as $\mathbf{Y}_{\mathbf{X}^{\perp}}=\mathbf{Q Y}$, with $\mathbf{Q}=\mathbf{I}-\mathbf{P}$. It is clear that the main result of this algorithm is the computation of a component of heart rate linearly related to respiration denoted by $\mathbf{Y}_{\mathbf{X}}$, and a residual component related to mechanisms other than respiration denoted by $\mathbf{Y}_{\mathbf{X}^{\perp}}$. Note that the latter will also contain non-linear influences of the respiration, which are not characterized by this approach.

The components derived using the wavelet decomposition of the respiration will be denoted by $\mathbf{Y}_{\mathbf{X}_{w}}$ and $\mathbf{Y}_{\mathbf{X}_{w}^{\perp}}$, and the components obtained after using $\mathbb{V}_{s}$, will be denoted by $\mathbf{Y}_{\mathbf{X}_{s}}$ and $\mathbf{Y}_{\mathbf{X}_{s}^{\perp}}$. From each component, the frequency domain parameters will be calculated, namely, the absolute and normalized powers in the low and high frequency bands, and the sympathovagal balance. The low frequency power $(L F)$ is defined in the range between $0.04 \mathrm{~Hz}$ an $0.15 \mathrm{~Hz}$, and the high frequency power $(H F)$ is computed in the range between $0.15 \mathrm{~Hz}$ and half of the mean heart rate for each segment. Note that in this study, the limits for the HF band are not modified using the respiratory frequency, as implemented in [1]. The reason for this is that the HF modification requires a peaked respiratory spectrum, which might not be the case for multiple recorded respiratory signals. Consequently, by keeping the 
limits fixed all the segments can be included in the analysis.

The normalized LF power is then defined as $L F_{n}=$ $L F /(L F+H F)$, and the sympathovagal balance as $L F / H F$. The subindices $s$ and $w$ will be used to indicate the subspace, namely the original signal or its wavelet decomposition, respectively.

\subsection{Statistical Test}

The differences between the frequency features of the heart rate components for different stress levels will be evaluated using the Friedman test for repeated measures. Since 4 different stress levels will be evaluated, a multicomparison test will be performed with Bonferroni correction equal to $\alpha / 6=0.008$ and $\alpha=0.05$.

\section{Results and Discussion}

The heart rate signal was decomposed using two different subspaces: the wavelet decomposition of the respiratory signal [2], and the original respiratory signal [3]. From each decomposition, two components were derived and they were characterized by means of the well-known frequency domain parameters of HRV. The median and median absolute deviation (MAD) of these parameters for each stress phase are indicated in Table 1, and the normalized powers in the LF band for each component and each stress phase are depicted in Figure 1. The figure also indicates the normalized LF power of the original HRV signal, $L F_{n, o r}$ in order to illustrate the added value of the heart rate decomposition in the analysis of stress.

It is clear that the power of the respiratory components in the low frequency band $\left(L F_{n, w}\right.$ and $\left.L F_{n, s}\right)$ is higher during $\mathrm{BL}$, which suggests that during this phase, the respiratory rate goes below the upper limit of the classical LF band. This, in the classical HRV analysis, could result in an overestimation of the sympathetic activity, as can be observed from the normalized LF power of the original HRV signal, namely $L F_{n, o r}$. These results are in agreement with those reported in [1], where the respiratory rate was found within the LF band for different cases, especially during BL. At this point, it is clear that the quantification of the respiratory influences on the heart rate leads to a more accurate quantification of the ANS modulation.

From the normalized frequency parameters it is also possible to conclude that stress has a strong effect on the linear interactions between respiration and heart rate. As can be seen from Figure 1, the residual components are not different between the phases, which confirms the hypothesis outlined in [1], where only the respiratory information might be enough for the detection of stress. However, when looking at the power in the HF band for the residual components (see Table 1), all phases are significantly dif- ferent from BL. These results are in agreement with those reported in [2], where the residual heart rate was found to have the highest discriminative power for stress detection. Nevertheless, in [2], no difference was found between the respiratory component during relax and stress. One possible explanation for this discrepancy is that this study and the one in [2] deal with two different types of stress, namely, emotional stress and mental stress, respectively. Consequently, no strong conclusions can be drawn about the residual heart rate during different types of stress until they are analysed simultaneously.

Another important point to keep in mind is that only linear respiratory influences were considered in this analysis. Therefore, it might be that nonlinear interactions are still contained in the residual components of HRV and that they are different for each stress phase, as suggested in [5]. These nonlinear interactions can be responsible for the significant difference in the HF power of the residual components, $\mathbf{Y}_{\mathbf{X}^{\perp}}$, between BL and all stress phases. An increased $\mathrm{HF}$ can also be associated to other vagally mediated effects different from respiration but this can only be confirmed by means of a non linear approach.

When evaluating all frequency domain parameters, no significant difference was found between the parameters obtained with both approaches. Hence, no added value is introduced in the quantification of the cardiorespiratory interactions when the wavelet decomposition is used. This is important because the computational requirements can be reduced by simplifying the construction of the respiratory subspace.

\section{Conclusions}

This study showed that linear respiratory dynamics account for most of the differences in HRV that appear at different emotional stress phases. This was observed after decomposing the heart rate signal using orthogonal subspace projections with two different respiratory subspaces. The results obtained with both subspaces indicated that no added value in the differentiation of stress was achieved with the wavelet decomposition. As a result, a simpler algorithm was proven useful for the separation of respiratory influences from the heart rate, and for the discrimination between relax and stress. Nevertheless, only linear cardiorespiratory interactions were analysed, hence, further analysis needs to be done on the heart rate dynamics that remain after removing lineal respiratory influences.

\section{Acknowledgements}

This work is supported by: OSA+. iMinds Medical Information Technologies: ICON HBC.2016.0167. European Research Council: The research leading to these results has received funding from the European Research 
Table 1. Median \pm MAD of the frequency domain parameters for each stress phase, and derived from the respiratory component $\mathbf{Y}_{\mathbf{X}}$ and the residual component $\mathbf{Y}_{\mathbf{X}^{\perp}}$.

\begin{tabular}{|l|cccc|cccc|}
\hline HRV & \multicolumn{4}{|c|}{ Wavelet-based approach $(w)$} & \multicolumn{4}{c|}{ Original respiratory signal $(s)$} \\
\cline { 2 - 9 } index & BL & ST & SA & VE & BL & ST & SA \\
\hline \hline $\mathbf{Y}_{\mathbf{X}}$ & & & & & & \\
$L F$ & $52.78 \pm 25.1$ & $14.31 \pm 92.2^{*}$ & $11.90 \pm 8.7^{*}$ & $7.42 \pm 4.7^{*}$ & $50.17 \pm 28.4$ & $8.36 \pm 11.2^{*}$ & $9.76 \pm 7.4^{*}$ & $5.09 \pm 5.2^{*}$ \\
$H F$ & $75.83 \pm 27.8$ & $68.54 \pm 26.6$ & $43.02 \pm 19.3^{*}$ & $53.45 \pm 28.1$ & $66.08 \pm 29.8$ & $56.58 \pm 26$ & $38.27 \pm 18.8^{*}$ & $53.31 \pm 27.7$ \\
$L F_{n}$ & $0.40 \pm 0.16$ & $0.20 \pm 0.12^{*}$ & $0.25 \pm 0.13^{*}$ & $0.10 \pm 0.08^{*}$ & $0.43 \pm 0.17$ & $0.18 \pm 0.12^{*}$ & $0.22 \pm 0.13^{*}$ & $0.10 \pm 0.08^{*}$ \\
$L F$ & $0.67 \pm 0.79$ & $0.25 \pm 0.23^{*}$ & $0.34 \pm 0.32^{*}$ & $0.11 \pm 0.13^{*}$ & $0.78 \pm 0.95$ & $0.22 \pm 0.25^{*}$ & $0.28 \pm 0.24^{*}$ & $0.11 \pm 0.16^{*}$ \\
\hline \hline$F F$ & & & & & & \\
$\mathbf{Y}_{\mathbf{X}^{\perp}}$ & $53.77 \pm 22.7$ & $58.04 \pm 92.5$ & $91.27 \pm 21.0^{*}$ & $76.82 \pm 21.8$ & $58.78 \pm 23.0$ & $60.34 \pm 31.0$ & $94.77 \pm 21.3^{*}$ & $77.83 \pm 24.3$ \\
$L F$ & $32.88 \pm 14.2$ & $45.68 \pm 20.6^{*}$ & $58.01 \pm 15.4^{*}$ & $64.98 \pm 17.2^{*}$ & $35.71 \pm 15.0$ & $52.76 \pm 20.9^{*}$ & $60.54 \pm 15.7^{*}$ & $67.33 \pm 18.9^{*}$ \\
$H F$ & $0.64 \pm 0.10$ & $0.64 \pm 0.12$ & $0.61 \pm 0.08$ & $0.56 \pm 0.10^{*}$ & $0.63 \pm 0.10$ & $0.57 \pm 0.11$ & $0.60 \pm 0.09$ & $0.53 \pm 0.10$ \\
$L F_{n}$ & $1.80 \pm 0.66$ & $1.80 \pm 0.92$ & $1.59 \pm 0.56$ & $1.29 \pm 0.51^{*}$ & $1.72 \pm 0.64$ & $1.33 \pm 0.59$ & $1.51 \pm 0.59$ & $1.16 \pm 0.51$ \\
\hline$L F$ &
\end{tabular}

${ }^{*}$ Significant difference with respect to baseline (BL) with a $p$-value $<0.008$.

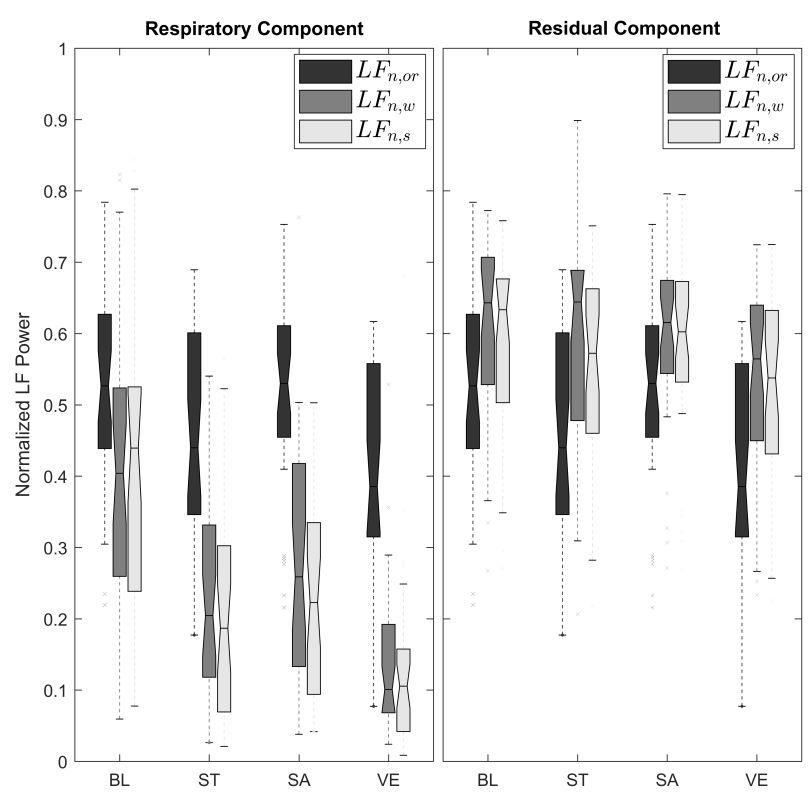

Figure 1. Normalized powers of the heart rate and its two components in the LF band. or stands for the original HRV signal, $w$ and $s$ refer to the subspace, namely $\mathbb{V}_{w}$ and $\mathbb{V}_{s}$, respectively. No significant differences were found between the wavelet-based approach and the one based on the original respiratory signal.

Council under the European Union's Seventh Framework Programme (FP7/2007-2013) / ERC Advanced Grant: BIOTENSORS (n 339804). TIN2014-53567-R (MINECO, Spain), T96 (Government of Aragon and European Social Fund), CIBER-BBN (Instituto de Salud Carlos III and FEDER). This paper reflects only the authors' views and the Union is not liable for any use that may be made of the contained information. Carolina Varon is a postdoctoral fellow of the Research Foundation-Flanders (FWO).

\section{References}

[1] Hernando A, Lázaro J, Gil E, Arza A, Garzón JM, LópezAntón R, de la Cámara C, Laguna P, Aguiló J, Bailón R. Inclusion of respiratory frequency information in heart rate variability analysis for stress assessment. IEEE journal of biomedical and health informatics 2016;20(4):1016-1025.

[2] Widjaja D, Caicedo A, Vlemincx E, Van Diest I, Van Huffel S. Separation of respiratory influences from the tachogram: a methodological evaluation. PloS one 2014;9(7):e101713.

[3] Varon C, Caicedo A, Testelmans D, Buyse B, Van Huffel S. A novel algorithm for the automatic detection of sleep apnea from single-lead ecg. IEEE Transactions on Biomedical Engineering 2015;62(9):2269-2278.

[4] Mateo J, Laguna P. Analysis of heart rate variability in the presence of ectopic beats using the heart timing signal. IEEE Transactions on Biomedical Engineering 2003;50(3):334343.

[5] Kontaxis S, Lázaro J, Hernando A, Arza A, Garzón JM, Gil E, Laguna P, Aguiló J, Bailón R. Mental stress detection using cardiorespiratory wavelet cross-bispectrum. In Computing in Cardiology Conference (CinC), 2016. IEEE, 2016; 725-728.

Address for correspondence:

Carolina Varon

ESAT/STADIUS/KU Leuven

Kasteelpark Arenberg 10, bus 2446, 3001 Leuven, Belgium. carolina.varon@esat.kuleuven.be 